\title{
Spectroscopic Observations of Hundreds of Star Formation Regions in M33
}

\author{
Xu Kong, Ning Hu and Fuzhen Cheng \\ CAS Key Laboratory for Research in Galaxies and Cosmology, Department of Astronomy, \\ University of Science and Technology of China, Hefei, Anhui 230026, China \\ email: xkong@ustc.edu.cn
}

\begin{abstract}
Star-formation regions in nearby galaxies provide an excellent laboratory to study star formation processes, evolution of massive stars and the properties of the surrounding interstellar medium. A wealth of information can be obtained from their spectral analysis of the emission lines and the stellar continuum. Considering these, we proposed a long-term project "Spectroscopic Observations of the Star Formation Regions in Nearby Galaxies". The primary goal of this project is to observe spectroscopy of star formation regions in 20 nearby galaxies, with the NAOC $2.16 \mathrm{~m}$ telescope and the Hectospec/MMT multifiber spectrograph. With the spectra of a large sample of star formation regions, combining multi-wavelength data from UV to IR, we can investigate, understand and quantify the dust extinction, star formation rate, metal abundance, and the two-dimensional distributions of stellar population properties of nearby galaxies. It will be important for a better understanding of galaxy formation. Here we report on the observations, data reduction, and analysis of the spectra of $\sim 400$ star formation regions in M33, via multifiber spectroscopy with Hectospec at the MMT.
\end{abstract}

Keywords. H II regions, galaxies: abundances, galaxies: individual (M33).

\section{Introduction and Observation}

The spatial-resolved spectroscopic data are very important for understanding the internal composition and kinematic characteristics of a galaxy, and therefore its formation, structure, and evolution. The IFU (Integral Field Unit) spectrograph is the most important tool for this kind of observations. Some systematic two-dimensional spectroscopic observations on galaxies were successively made recently, but most of these observations are limited on the central region of large galaxies or small galaxies, because the fields of view of most IFUs are relative small (Kong et al. 2014).

M33 (NGC 598) is one of the closest spiral galaxies of the Local Group. The proximity of M33, together with its large angular size (optical size $53^{\prime} \times 83^{\prime}$ ), and its intermediate inclination $(i=53 \mathrm{deg})$, allows detailed studies of its stellar populations and ionized nebulae. M33 is a galaxy rich in star formation regions (H II regions). Several catalogs of the M33 $\mathrm{H}$ in regions have been published. Their spectroscopy was obtained since the 1970s, however, most of them are small and inhomogeneous.

To obtain a large, homogeneous set of spectra for HII regions in M33, we have used the MMT/Hectospec, its has a wide field of view (1 degree diameter field) and large number of fibers (300 in total) for multi-object spectroscopy. The targets for the multi-object spectroscopy were selected from narrow-band $\mathrm{H} \alpha$ image. M33 has more than $400 \mathrm{H} \mathrm{II}$ regions, the spectroscopic data were acquired in Oct. 9 (M33-A, red), Oct. 12 (M33-B, green), and Oct. 15 (M33-C, blue), 2013, as shown in Figure 1. The exposure time of each is $5400 \mathrm{~s}$. The MMT/Hectospecs 270 line $\mathrm{mm}^{-1}$ grating was used, which provides a dispersion of $1.2 \AA /$ pixel. Our spectra cover the wavelength range of $3650-9150 \AA$ at $\sim 5 \AA$ resolution.

\section{Data Reduction and Implications}

The spectra were reduced using the publicly available HSRED software (written by R. Cool). All observations were bias subtracted, overscan corrected, and trimmed. The science exposures were 

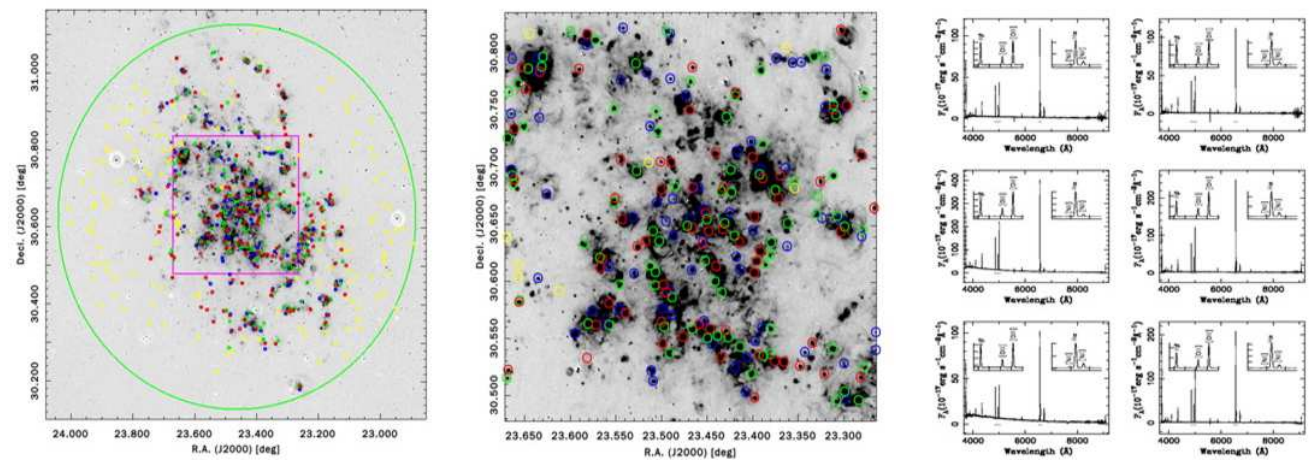

Figure 1. Left: Location of M33 H II regions and the sky background (yellow) observed with Hectospec, overlaid on the M33 Ha image. The green circle shows the Hectospec field. Middle: The M33 center region is expanded, most of bright $\mathrm{H}$ II regions were observed by MMT/Hectospec. Right: The example spectra of six star formation regions in M33.

flat fielded and combined together to eliminate cosmic rays, and the one-dimensional spectra were extracted and wavelength calibrated. In the right panel of Figure 1, as an example, we show the spectra of some H II regions. The emission-line fluxes were measured with the package SPLOT Of IRAF.

With the spectra of a large sample of star formation regions, combining multi-wavelength image data from UV to IR, we can investigate, understand and quantify the dispersion of the IRX (the total-IR to UV flux ratio) $-\beta$ relation and gradients and physical properties of galaxies.

Based on multi-wavelength photometry data, many work have found that individual H II regions of nearby galaxies have a large dispersion in the IRX - $\beta$ diagram (Mao et al.2012). Considering there have simple star formation histories and have strong emissiom lines, H II regions in nearby galaxies are ideal targets to quantify the nature of the dispersion for galaxies in the IRX $-\beta$ diagram. Combining multi-wavelength data from UV to IR, we will investigate, understand and quantify the nature of the deviation from the starbursts IRX $-\beta$ correlation (Kong et al.2004). It will be important for a better understanding of the interaction of dust and radiation in dusty star-forming galaxies.

The spectra of those H II can also be used to study the radial metallicity gradients of galaxies (with much larger radius), calibrate metallicity estimation with different method (such as strong line method and $T_{e}$ method), investigate the radial distribution of dust properties, stellar populations and star formation.

This study has used the observational time of the $2.16 \mathrm{~m}$ telescope at the Xinglong station of the National Astronomical Observatories of China, and the observational time of the MMT telescope obtained via the TAP program.

\section{References}

Kong, X., Charlot, S., Brinchmann, J., \& Fall, S. M. 2004, MNRAS, 349, 769

Kong, X., Lin, L., Li, J.-r., et al. 2014, ChA\&A, 38, 427

Mao, Y.-W., Kennicutt, R. C., Jr., Hao, C.-N., Kong, X., \& Zhou, X. 2012, ApJ, 757, 52 